\title{
PROPOSTA DE UM MODELO PARA CÁLCULO DIRETO DA LATITUDE EM FUNÇÃO DAS COORDENADAS CARTESIANAS
}

\author{
Proposing of a Model for Calculus Direct of \\ Latitude by the Cartesian Coordinates
}

Claudionor Ribeiro da Silva

crs@ig.ufu.br

Universidade Federal de Uberlândia

\section{Resumo}

Os avanços tecnológicos ocorridos na Geodésia Espacial têm facilitado a obtenção de coordenadas geodésicas em distintos sistemas de referência. A conversão dessas coordenadas, de um sistema para outro, é uma tarefa rotineira, especialmente em projetos de engenharia e cadastro. Contudo, o modelo comumente utilizado para converter as coordenadas cartesianas em geodésicas, faz uso de um procedimento iterativo, ao determinar a latitude, o que insere um erro no módulo dessa coordenada. Nesse contexto, um modelo é proposto para determinação da latitude, diretamente, a partir das coordenadas cartesianas. Os resultados obtidos mostram as vantagens e desvantagens do modelo proposto, quando comparados aos resultados dos modelos iterativos.

Palavras-chave: Transformação de coordenadas, Sistemas de referência, Coordenadas Cartesianas. 


\section{Abstract}

Technological advances occurred in Geodesy have easier to obtain geodetic coordinates in different reference systems. The conversion of these coordinates from one system to another is a routine task, especially in engineering. However, the model commonly used to convert the Cartesian coordinates into geodetic uses an iterative procedure to determine latitude, which includes an error to coordinate. In this context, a model is proposed to determine the latitude directly from the Cartesian coordinates. The results show the advantages and disadvantages of the proposed model, when compared to the results of iterative models.

Keywords: Transformation of coordinates, System reference, Cartesian coordinates.

\section{Introdução}

As coordenadas de um determinado ponto na superfície terrestre são representadas de formas distintas, dependendo do sistema em que estão vinculadas. Os sistemas de coordenadas mais comuns são: Polares (ou Geodésicos), Retangulares / Cartesianos (ou Tridimensionais) e Plano-Retangulares (ou Plano Topográfico Local) (NADAL, 2006; DAL'FORNO et al., 2010).

No sistema de coordenadas Geodésicas, um ponto é representado pelo terno: Latitude, Longitude e Altitude ( $\phi, \lambda, \mathrm{h})$; no Retangular/ Cartesiano, pelo terno (X, Y, Z), com origem no centro da Terra (Geocêntrico); e, no Plano-Retangular/Local, pelo terno (x,y,z), com origem em um ponto qualquer na superfície terrestre, pertencente ao plano topográfico.

A transformação entre coordenadas Geodésicas $(\phi, \lambda, h)$ e Cartesianas $3 \mathrm{D}(\mathrm{X}, \mathrm{Y}, \mathrm{Z})$ foi extensivamente estudada atualmente, chamando a atenção de Geodesistas, engenheiros aeroespaciais e outros. Contudo, o assunto não foi completamente explorado, ocorrendo até algumas repetições de ideias (FEATHERSTONE e CLAESSENS, 2008). Segundo Seemkooei (2002), a conversão de coordenadas geodésicas em cartesianas é uma das mais importantes tarefas em Geodésia computacional.

$\mathrm{Na}$ execução de alguns projetos de engenharia e de cadastros, a transformação de coordenadas é indispensável. Em posicionamentos GNSS (Global NavigationSatellite System - atualmente operam o GPS (EUA) e o GLONASS (Rússia) e futuramente o COMPASS-BEIDU 
(China) e o GALILEO (União Europeia)), bastante usado na atualidade, esse procedimento é necessário, pois esses sistemas de navegação coletam, inicialmente, as coordenadas cartesianas $3 \mathrm{D}$ e, posteriormente, são convertidas em geodésicas.

O procedimento para transformar coordenadas geodésicas em cartesianas 3D é relativamente simples, com modelos matemáticos diretos, contudo, o processo inverso não é trivial, podendo ser direto (PAUL, 1973;OZONE, 1985 e SOFAIR 1993) ou iterativo o cálculo da latitude (FOK e IZ, 2003; BURTCH (2006); GERDAN e DEAKIN (1999); CLYNCH, 2006 e FEATHERSTONE e CLAESSENS, 2008). O modelo usado na maioria dos softwares comerciais, para cálculo da latitude, é o iterativo, por ser classicamente usado e por ser um dos mais antigos, especialmente o modelo descrito por Heiskanen e Moritz (1967).

O procedimento iterativo retorna uma aproximação do valor real, sendo, portanto, inerente a erros (FRANCOIS, 2000). Os erros são devidos ao limiar usado para parada do processo iterativo e ao truncamento das séries finitas usadas na determinação das funções trigonométricas empregadas no modelo. Assim, o módulo da latitude calculada depende do método empregado, podendo variar de um software para outro.

Segundo Gerdan e Deakin (1999), os métodos diretos apresentam problemas no cálculo de baixas Latitudes, mas reduzem o tempo de processamento. Nesse contexto, é proposto neste estudo um modelo matemático mais simples que aqueles encontrados na literatura. $\mathrm{O}$ modelo proposto define diretamente a latitude de um ponto, a partir do terno de coordenadas cartesianas e dos parâmetros da superfície de referência (elipsoide de revolução).

\section{Sistemas De Coordenadas Geodésicas}

A determinação de um ponto na superfície da Terra se caracteriza pelo cálculo de suas coordenadas em relação a um sistema de referência, estabelecido de tal forma que todos os pontos tenham uma posição unívoca nesse sistema (SILVA, 2011). A superfície de referência deve ser posicionada e orientada em relação ao globo terrestre.

No sistema de coordenadas geodésicas, o modelo matemático, adotado como superfície de referência é o elipsoide. Essa superfície 
matemática é dividida em círculos paralelos ao equador (paralelos), e em elipses perpendiculares aos paralelos e passantes pelos pólos terrestres (meridianos), como mostra a Figura 1.

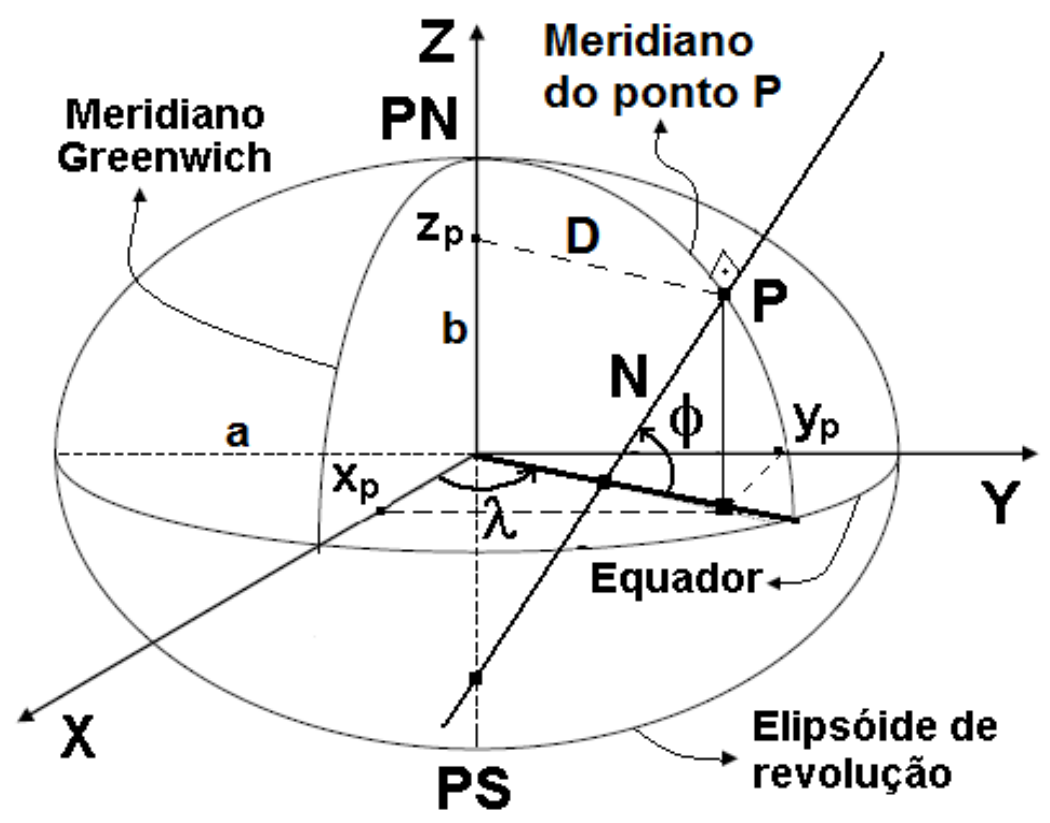

Figura 1. Exemplo hipotético de um sistema geodésico de referência.

Considerando um ponto P, localizado na superfície do elipsoide, um único par de coordenadas geodésicas (latitude e longitude) o definirá. Contudo, se esse ponto estiver na superfície da Terra, é necessária a definição de uma terceira coordenada, a altitude. Dependendo da superfície de referência utilizada, a altitude é denominada: geométrica (h) ou ortométrica (H); se a superfície de referência é o modelo matemático, o elipsoide, a altitude é geométrica, medida sobre a normal ao elipsoide, partindo da superfície do elipsoide até o ponto localizado na superfície da Terra. Entretanto, se a superfície de referência é o modelo físico, o Geoide (superfície física que mais se aproxima da forma da Terra), a altitude é dita ortométrica, e é medida sobre a vertical do lugar, do geoide até o ponto na superfície da Terra, como ilustra a Figura 2. 


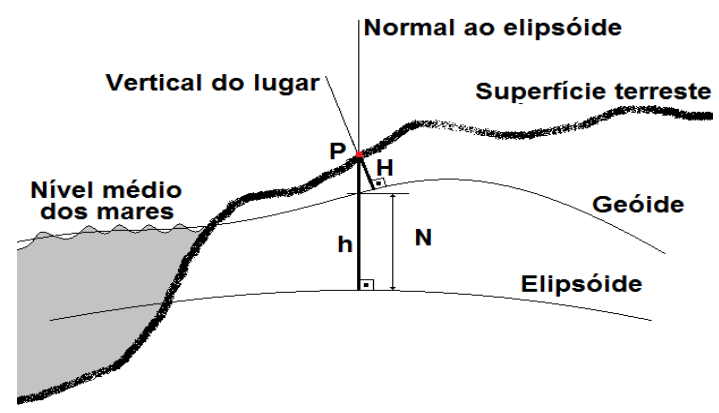

Figura 2. Altitudes de um ponto na superfície terrestre.

A diferença entre essas altitudes é denominada de "ondulação geoidal” e é representada por N. Deve-se ter cuidado para não confundir essa variável com a ordenada (N) do sistema UTM, mostrada anteriormente, ou com a grande normal $(\mathrm{N})$, que será apresentada a seguir.

A latitude geodésica $(\phi)$ é definida como o ângulo entre a normal ao elipsoide de referência, passante pelo ponto $\mathrm{P}$, localizado na superfície da Terra, e sua projeção no plano equatorial; tem origem no Equador e é positiva na direção Equador-Norte (de $\circ \mathrm{a}+90^{\circ}$ ) e negativa na Equador-Sul (de o a $-90^{\circ}$ ).

A longitude geodésica ( $\lambda$ ) é ângulo diedro formado pelos planos do meridiano de Greenwich e do meridiano que passa por P. Esse ângulo é contado sobre o plano do equador e tem por origem o meridiano de Greenwich, sendo positivo na direção Greenwich-Leste $\left(0 \mathrm{a}+180^{\circ}\right) \mathrm{e}$ negativo Greenwich-Oeste $\left(\mathrm{o}\right.$ a $\left.-180^{\circ}\right)$.

\section{Sistemas De Coordenadas Cartesianas Tridimensionais}

O sistema de coordenadas cartesianas tridimensionais é composto por três eixos cartesianos (X,Y,Z), ortogonais entre si, com origem no centro da superfície de referência. Em sistemas de referência, a origem dos eixos coordenados $(\mathrm{X}, \mathrm{Y}, \mathrm{Z})$ pode coincidir com o geocentro (centro de massa da Terra), caracterizando os sistemas Geocêntricos/Globais (para uso global). A não coincidência com o geocentro define os sistemas locais (para atender uma certa região do globo).

Nesses sistemas, o plano XZ é o plano do meridiano de Greenwich (origem das longitudes), e o plano XY é o plano equatorial da superfície de referência (origem das latitudes). O eixo das abscissas (X) está na di- 
reção da intersecção do plano XZ com o XY, com sentido positivo na direção da longitude $0^{\circ}$. A ordenada $(\mathrm{Y})$ está direcionada ortogonalmente e à Leste de $\mathrm{X}$, jacente ao plano XY. O eixo $\mathrm{Z}$ coincide com o eixo rotacional da superfície de referência, com sentido positivo na direção Norte (GERDAN e DEAKIN, 1999; GEMAEL e ANDRADE, 2004). A configuração desse sistema é ilustrada, juntamente com o sistema polar, na Figura 1.

\section{Transformação de Coordenadas}

A transformação de coordenadas é na realidade a conversão de coordenadas entre dois sistemas de coordenadas distintos, tais como: de um sistema Cartesiano para outro Cartesiano não coincidente, de Cartesiano para Esférico, de Polar para Cilíndrico e de Elíptico para Cartesiano. Em Geodésia, são mais comuns as transformações entre dois sistemas Cartesianos ou de coordenadas cartesianas para coordenadas geodésicas (vice-versa), definidas no elipsoide.

Em trabalhos geodésicos, ou de engenharia, é mais comum a necessidade de outro tipo de transformação, a de coordenadas geodésicas de um sistema A para coordenadas geodésicas de um sistema B. O procedimento comumente usado para realizar essa transformação demanda uma fase intermediária, compreendida por três passos: a) a transformação das coordenadas geodésicas para o sistema retangular, vinculado ao A; b) a transformação das coordenadas cartesianas de A para o sistema Cartesiano B; e c) o retorno das coordenadas Cartesianas para geodésicas, porém já no sistema $\mathrm{B}$.

No primeiro passo (a), as coordenadas geodésicas $(\phi, \lambda, h)$ são transformadas em coordenadas cartesianas $(\mathrm{X}, \mathrm{Y}, \mathrm{Z})$, por meio dos modelos matemáticos apresentados na equação ı (SEEBER, 1993). São fórmulas de manipulação simples, direta e facilmente programáveis em softwares.

$$
\begin{gathered}
\mathrm{X}=(\mathrm{N}+\mathrm{h}) \cos \phi \cos \lambda \\
\mathrm{Y}=(\mathrm{N}+\mathrm{h}) \cos \phi \operatorname{sen} \lambda \\
\mathrm{Z}=\left[\left(1-\mathrm{e}^{2}\right) \mathrm{N}+\mathrm{h}\right] \operatorname{sen} \phi
\end{gathered}
$$

em que, N é a grande normal, dada pela equação 2 .

$$
\mathrm{N}=\frac{\mathrm{a}}{\sqrt{1-\mathrm{e}^{2} \operatorname{sen}^{2} \phi}}
$$

e "e" é a excentricidade da elipse ou meridiano (equação 3). 


$$
\mathrm{e}=\frac{\sqrt{\mathrm{a}^{2}-\mathrm{b}^{2}}}{\mathrm{a}}
$$

A transformação de coordenadas cartesianas tridimensionais, entre dois sistemas Cartesianos A e B (passo b), pode ser simples quando se trata de sistemas paralelos e de mesma escala (equação 4), como WGS84 e SAD69 (MONICO,2000), que consiste apenas de 3 translações $\left(\mathrm{T}_{\mathrm{x}}, \mathrm{T}_{\mathrm{y}}, \mathrm{T}_{\mathrm{z}}\right)$.

$$
\begin{aligned}
& \mathrm{X}_{\mathrm{a}}=\mathrm{X}_{\mathrm{b}}+\mathrm{T}_{\mathrm{x}} \\
& \mathrm{Y}_{\mathrm{a}}=\mathrm{Y}_{\mathrm{b}}+\mathrm{T}_{\mathrm{y}} \\
& \mathrm{Z}_{\mathrm{a}}=\mathrm{Z}_{\mathrm{b}}+\mathrm{T}_{\mathrm{z}}
\end{aligned}
$$

Todavia, se não são paralelos e não têm a mesma escala, além das translações (T), devem ser levados em consideração o fator de escala (k) e a matriz de rotações $\mathrm{R}(\delta, \Psi, \gamma)$ em torno dos três eixos cartesianos, como mostrado na equação 5. Exemplos desses modelos são os de Molodensky e Helmert/Bursa-Wolf (MONICO, 2000).

$$
\mathrm{X}_{\mathrm{a}}=\mathrm{kR}(\delta, \psi, \gamma) \mathrm{X}_{\mathrm{b}}+\mathrm{T}
$$

Nesse modelo, $\mathrm{Xa}$ e $\mathrm{Xb}$ são vetores compostos pelos ternos (X,Y,Z) de pontos $\mathrm{P}$, localizados nos sistema Cartesianos A e B, respectivamente.

A transformação realizada no terceiro passo (c) não é tão simples como aquelas realizadas nos passos anteriores. Essa etapa pode ser determinada de forma direta ou iterativa. Com base nos modelos matemáticos tradicionais (equação 6), propostos por Heiskanen e Moritz (1967), a latitude é determinada iterativamente (em que $\mathrm{N}(\phi)$ ), o que demanda maior esforço computacional, além do erro inerente ao processo.

$$
\left\{\begin{array}{c}
\lambda=\tan ^{-1}\left(\frac{\mathrm{Y}}{\mathrm{X}}\right) \\
\phi=\tan ^{-1}\left(\frac{\mathrm{Z}}{\sqrt{\mathrm{X}^{2}+\mathrm{Y}^{2}}}\left(1-\frac{\mathrm{e}^{2} \mathrm{~N}}{\mathrm{~N}+\mathrm{h}}\right)^{-1}\right) \\
\mathrm{h}=\left(\frac{\sqrt{\mathrm{X}^{2}+\mathrm{Y}^{2}}}{\cos \phi}\right)-\mathrm{N}
\end{array}\right.
$$


Mesmo com esse problema intrínseco, vários países ainda fazem uso desse modelo, como o Instituto Geográfico Nacional Francês, que adota o algoritmo iterativo que faz uso do modelo da equação 6 (FRANCOIS, 2000).

Os modelos diretos, usados nessa fase (c), determinam a Latitude $(\phi)$ diretamente, com base no terno Cartesiano (X, Y, Z). Existem alguns modelos apresentados na literatura, mas são geralmente equações complexas, como aquelas propostas por Paul (1973) e Ozone (1985). Os modelos diretos propostos por Paul (1973) e Ozone (1985) são compostos pela integração de sete e dez equações, respectivamente (GERDAN e DEAKIN, 1999).

\section{Modelo Proposto}

Frente aos problemas de arredondamento/morosidade do modelo iterativo e à complexidade das equações dos modelos diretos, ambos usados no cálculo da latitude, é proposto neste estudo um modelo simples para cálculo direto dessa medida. O modelo proposto é fundamentado nos parâmetros do elipsoide, semi-eixo maior "a" e semi-eixo menor "b", e nas coordenadas geodésicas e cartesianas (retangulares) de um ponto $\mathrm{P}$, jacente sobre a superfície do elipsoide, como é ilustrado na Figura 1.

Em uma primeira fase, considere a elipse que pertence ao plano ZY (Figura 3).

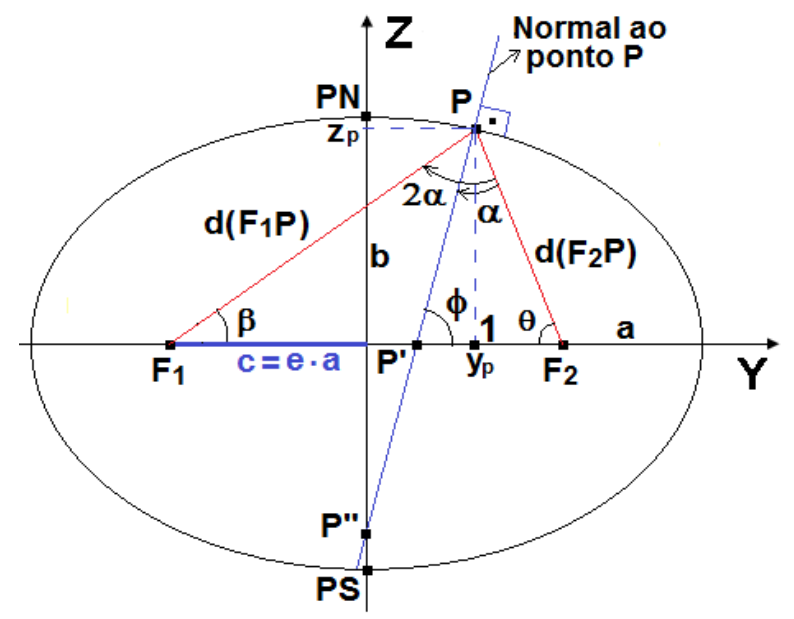

Figura 3. Elipse jacente no plano ZY. 
Das propriedades geométricas da elipse, três, usadas na demonstração do modelo proposto, são destacadas: a) a distância focal (c) da origem do sistema $(\mathrm{O}, \mathrm{O})$ a um dos focos (F1 ou F2) é igual ao produto da excentricidade da elipse (e) pelo semi-eixo maior (a); b) a reta normal ao elipsoide, em qualquer ponto $\mathrm{P}(\mathrm{Xp}, \mathrm{Yp}, \mathrm{Zp})$, é a bissetriz do ângulo $(2 \alpha)$ formado pelas duas direções PF1 e PF2 (ALHANATI, 2011); e c) a soma das distâncias medidas dos focos 1 e 2, até o ponto $P$, é igual a duas vezes o semi-eixo maior (a), como exposto na equação 7.

$$
\mathrm{d}\left(\mathrm{F}_{1} \mathrm{P}\right)+\mathrm{d}\left(\mathrm{F}_{2} \mathrm{P}\right)=2 \mathrm{a}
$$

Com base no triângulo ( $\left.\mathrm{F}_{1} \mathrm{PF} 2\right)$, formado na elipse ilustrada na Figura 3, em conceitos da trigonometria plana e em propriedades geométricas da elipse, são determinadas as equações 8, 9, 10, 11, 12 e 13, demonstradas a seguir.

Do triângulo retângulo $\mathrm{PF}_{2}$ :

$$
\begin{array}{r}
d\left(F_{2} P\right)=\sqrt{Z_{p}^{2}+\left(c-Y_{p}\right)^{2}} \\
\theta=\cos ^{-1}\left(\frac{c-Y_{p}}{\sqrt{Z_{p}^{2}+\left(c-Y_{p}\right)^{2}}}\right)
\end{array}
$$

A partir das equações 7 e 8:

$$
d\left(F_{1} P\right)=2 a-\sqrt{Z_{p}^{2}+\left(c-Y_{p}\right)^{2}}
$$

Do triângulo retângulo $\mathrm{PF}_{1}$ 1:

$$
\beta=\tan ^{-1}\left(\frac{Z_{p}}{c+Y_{p}}\right)
$$


Do triângulo escaleno $\mathrm{PF}_{1} \mathrm{~F}_{2}$, e das equações 9 e 11:

$$
\left\{\begin{array}{c}
2 \alpha=\pi-(\theta+\beta) \\
\alpha=\frac{\pi-\cos ^{-1}\left(\frac{c-Y_{p}}{\sqrt{Z_{p}^{2}+\left(c-Y_{p}\right)^{2}}}\right)-\tan ^{-1}\left(\frac{Z_{p}}{c+Y_{p}}\right)}{2}
\end{array}\right.
$$

Por fim, o modelo proposto é obtido a partir do triângulo escaleno PF2P':

$$
\phi=\pi-(\theta+\alpha)
$$

Partindo do caso particular mostrado na Figura 3, em que a elipse está sobre o plano ZY, e generalizando para um ponto qualquer na superfície do elipsoide, como P mostrado na Figura 1, o valor da distância $\mathrm{D}$, que era igual a Yp, no caso particular, passa a ser função de $\mathrm{D}(\mathrm{Xp}, \mathrm{Yp})$, no caso geral, como mostra a equação 14 .

$$
D=\sqrt{\mathrm{X}_{\mathrm{p}}^{2}+\mathrm{Y}_{\mathrm{p}}^{2}}
$$

Substituindo (9), (12) e (14), em (13), e atribuindo o valor de c = ea, tem-se que:

$\phi=\frac{1}{2}\left(\pi+\tan ^{-1}\left(\frac{Z_{p}}{e a+\sqrt{X_{p}^{2}+Y_{p}^{2}}}\right)-\cos ^{-1}\left(\frac{e a-\sqrt{X_{p}^{2}+Y_{p}^{2}}}{\sqrt{Z_{p}^{2}+\left(e a-\sqrt{X_{p}^{2}+Y_{p}^{2}}\right)^{2}}}\right)\right)(15)$

Para testar o modelo, foram realizados seis experimentos usando tanto o modelo proposto quanto o modelo apresentado em SÁ (2011), denominado de "Geocar". O Geocar foi escolhido por ser um modelo que determina a latitude e altitude por um processo iterativo (equação $6)$, diferente do modelo proposto (direto), e por ter sido desenvolvido em meio acadêmico, em que existe maior preocupação com os parâmetros acurácia/precisão, se comparado com softwares comerciais.

Para manter um padrão de comparação, as coordenadas dos pontos 
analisados foram escolhidas em coordenadas geodésicas e posteriormente convertidas para cartesianas, usando uma rotina desenvolvida em Matlab7b. Assim, essas coordenadas cartesianas foram submetidas aos dois modelos, sendo convertidas para coordenadas geodésicas novamente. Os resultados são comparados com as coordenadas geodésicas escolhidas inicialmente.

\section{Resultados e Discussões}

Para analisar os resultados, foram realizados experimentos com coordenadas de cinco pontos escolhidos estrategicamente, com coordenadas cartesianas positivas e/ou negativas, denominadas neste estudo de coordenadas reais, como mostra a Tabela 1. Essas coordenadas são referenciadas ao SIRGAS, que usa o Elipsoide GRS80 (GeodeticReference System 1980: semi-eixo maior $=6378,137 \mathrm{~m}$ e achatamento $=$ $1 / 298,257222101$ ) como superfície de referência.

Tabela ı. Coordenadas geodésicas e cartesianas dos pontos analisados

\begin{tabular}{|c|c|}
\hline Latitude $\left({ }^{\circ}\right)$ & Longitude $\left({ }^{\circ}\right)$ \\
\hline $25^{\circ} 25^{\prime} 25,000000^{\prime \prime}$ & $-25^{\circ} 25^{\prime} 25,000000^{\prime \prime}$ \\
\hline$-25^{\circ} 25^{\prime} 25,000000^{\prime \prime}$ & $120^{\circ} 25^{\prime} 25,000000^{\prime \prime}$ \\
\hline ০০০০০'০,০০3240" & $89^{\circ} 59 ' 59,996760 "$ \\
\hline ০০০০০'০,০০3240" & $179^{\circ} 59 ' 59,996760 "$ \\
\hline $89^{\circ} 59^{\prime} 59,995442 "$ & $45^{\circ} 00^{\prime} \circ 0,00000{ }^{\prime \prime}$ \\
\hline -89'59'59,995442" & 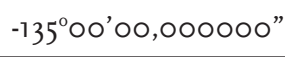 \\
\hline
\end{tabular}

\begin{tabular}{lccc}
\cline { 2 - 4 } & $\mathbf{X}(\mathrm{m})$ & $\mathrm{Y}(\mathrm{m})$ & $\mathrm{Z}(\mathrm{m})$ \\
\cline { 2 - 4 } $\mathbf{P}_{1}$ & 5205828,19299 & $-2474538,45167$ & 2721530,13193 \\
$\mathbf{P}_{2}$ & $-2918839,90856$ & 4970348,20832 & $-2721530,13193$ \\
$\mathbf{P}_{3}$ & 0,10000 & 6378137,000000 & 0,10000 \\
$\mathbf{P}_{4}$ & $-6378137,00000$ & 0,10000 & 0,10000 \\
$\mathbf{P}_{5}$ & 0,10000 & 0,10000 & 6356752,31400 \\
$\mathbf{P}_{6}$ & $-0,10000$ & $-0,10000$ & $-6356752,31400$ \\
\hline
\end{tabular}


Dos seis pontos analisados: $\mathrm{P} 1$ (ponto 1 ) pertence ao hemisfério Norte e P2 ao hemisfério Sul; os terceiro e quarto pontos ( $\mathrm{P}_{3}$ e $\mathrm{P} 4$ ) estão ligeiramente acima do plano do Equador, com baixos valores de Latitude, e muito próximos dos eixos $\mathrm{Y}$ e $-\mathrm{X}$, respectivamente; por outro lado, os pontos $\mathrm{P}_{5}$ e P6 apresentam altos valores de latitude, isto é, estão situados muito próximos aos pólos Norte e Sul, respectivamente. Pelo fato de não existir interferência nos resultados, a altitude geométrica (h) foi considerada nula em todos os experimentos.

Por se tratar de modelos que apresentam intrinsecamente funções trigonométricas, na forma direta ou inversa, os pontos de $\mathrm{P}_{3}$ a $\mathrm{P} 6$ foram coletados em regiões (próximo de o e de 90 graus) em que essas funções são bastante sensíveis às pequenas variações do ângulo. Para exemplificar essa sensibilidade, especialmente devido ao processo de arredondamento, o arco-cosseno de um ângulo com dez casas deci-

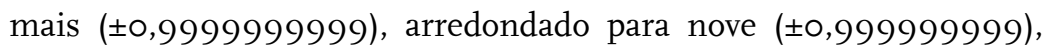
retorna uma diferença/erro, na superfície de uma esfera de raio 6371 $\mathrm{km}$ (raio médio da Terra), de aproximadamente 195 metros. Portanto, se esse mesmo arredondamento for realizado em um ângulo afastado dessa região crítica (o ou 90 graus), por exemplo, de 0,5555555555 para 0,555555555, a diferença/erro é de apenas 3,83 milímetros.

As coordenadas cartesianas transformadas pelos dois modelos, Geocar e o proposto, estão apresentadas na Tabela 2.

Tabela 2. Coordenadas geodésicas obtidas no processo de transformação.

\section{GEOCAR}

\begin{tabular}{cc} 
Latitude $\left(^{\circ}\right)$ & Longitude $\left(^{\circ}\right)$ \\
\hline $25^{\circ} 25^{\prime} 24,999997^{\prime \prime}$ & $-25^{\circ} 25^{\prime} 25,00 "$ \\
$-25^{\circ} 25^{\prime} 24,999999^{\prime \prime}$ & $120^{\circ} 25^{\prime} 25,00 "$ \\
$00^{\circ} 00^{\prime} 0,003256^{\prime \prime}$ & $89^{\circ} 59^{\prime} 59,996766^{\prime \prime}$ \\
$00^{\circ} 00^{\prime} 0,003256 "$ & $179^{\circ} 59^{\prime} 59,996766^{\prime \prime}$ \\
$89^{\circ} 59^{\prime} 59,993873^{\prime \prime}$ & $45^{\circ} 00^{\prime} 00,00^{\prime \prime}$ \\
$-89^{\circ} 59^{\prime} 59,993873^{\prime \prime}$ & $-135^{\circ} 00^{\prime} 00,00 "$ \\
\hline
\end{tabular}




\begin{tabular}{cc}
\hline \multicolumn{2}{c}{ MODELO PROPOSTO } \\
Latitude $\left(^{\circ}\right)$ & Longitude $\left(^{\circ}\right)$ \\
\hline $25^{\circ} 25^{\prime} 24,999997^{\prime \prime}$ & $-25^{\circ} 25^{\prime} 25,00^{\prime \prime}$ \\
$-25^{\circ} 25^{\prime} 24,999999^{\prime \prime}$ & $120^{\circ} 25^{\prime} 25,00^{\prime \prime}$ \\
$00^{\circ} 00^{\prime} 0,003032^{\prime \prime}$ & $89^{\circ} 59^{\prime} 59,996766^{\prime \prime}$ \\
$00^{\circ} 00^{\prime} 0,003032^{\prime \prime}$ & $179^{\circ} 59^{\prime} 59,996766^{\prime \prime}$ \\
$89^{\circ} 59^{\prime} 59,995442^{\prime \prime}$ & $45^{\circ} 00^{\prime} 00,00^{\prime \prime}$ \\
$-89^{\circ} 599^{\prime} 59,995442^{\prime \prime}$ & $-135^{\circ} 00^{\prime} 00,00 "$ \\
\hline
\end{tabular}

As diferenças medidas nos resultados do modelo proposto são da ordem dos milímetros, com módulos menores que 6,5mm. As coordenadas dos dois primeiros pontos ( $\mathrm{P}_{1}$ e $\mathrm{P}_{2}$ ) apresentaram diferenças iguais em ambos os modelos, Geocar e proposto. Contudo, nos demais pontos ( $\mathrm{P}_{3}, \mathrm{P}_{4}, \mathrm{P}_{5}$ e P6) ocorreram variações consideráveis, como exposto na Tabela 3. Ressalta-se que os valores apresentados na Tabela 3 foram obtidos a partir da transformação de segundos para milímetros, usando o raio da Terra igual a $6371 \mathrm{~km}$.

Tabela 3. Diferenças entre coordenadas reais (valores fixos) e calculadas (modelos) dadas em milímetros

\begin{tabular}{cc}
\hline GEOCAR & MODELO PROPOSTO \\
\hline 0,0926624 & 0,0926624 \\
0,0308875 & 0,0308875 \\
$-0,4941997$ & 6,4245958 \\
$-0,4941997$ & 6,4245958 \\
48,4624555 & 0,0000000 \\
48,4624555 & 0,0000000 \\
\hline
\end{tabular}

Nos pontos próximos ao equador ( $\mathrm{P}_{3}$ e $\mathrm{P}_{4}$ ), o modelo proposto apresentou coordenadas com maior módulo de diferenças $(6,42 \mathrm{~mm})$, se comparadas aos resultados do modelo Geocar. Todavia, em pontos próximos aos pólos ( $\mathrm{P}_{5}$ e P6), o modelo proposto apresentou maior acurácia $(48,46 \mathrm{~mm})$ em relação ao outro modelo analisado.

Nesses casos específicos dos pontos das regiões críticas, o modelo proposto apresentou vantagem nos pontos próximos aos pólos ( $\mathrm{P}_{5}$ e $\mathrm{P} 6)$, e piores resultados para pontos próximos ao equador $\left(\mathrm{P}_{3}, \mathrm{P}_{4}\right)$. Ob- 
servando o triângulo escaleno PF2P', ilustrado na Figura 3, nota-se que, quando um ponto $P$ se aproxima do equador, os ângulos $\alpha$ e $\theta$ tendem para $\circ$ (zero) e $-\pi$, respectivamente. Esse é o pior caso para o modelo, pois tanto a função arco-tangente de um ângulo próximo a zero quanto o arco-cosseno de um ângulo tendendo para $-\pi$ apresentam sensibilidade. Por outro lado, quando o valor da latitude aproxima de \pm 90 graus (pólos), o referido triângulo escaleno (Figura 3) apresenta os valores máximos conjuntamente para $\alpha$ e $\theta$, ilustrando a melhor condição para o modelo.

O modelo iterativo, usado no Geocar, é composto apenas de uma função arco-tangente, que é sensível ao aproximar de \pm 90 graus, o que explica o maior erro para pontos próximos aos pólos.

\section{Conclusão}

Os erros medidos neste estudo são chamados de "diferenças", porque, no processo de transformação de coordenadas geográficas para cartesianas, pode ter ocorrido algum erro, ainda que de arredondamento, uma vez que, nos modelos de conversão, também são utilizadas funções trigonométricas. Contudo, isso não invalida o método de comparação, uma vez que os valores de entrada (coordenadas já transformadas para cartesianas) em ambos os métodos, proposto e Geocar, são os mesmos (iguais).

Outra observação concreta é que as coordenadas geodésicas transformadas, de qualquer ponto $\mathrm{P}$, que não pertença às regiões próximas aos eixos coordenados, são similares para os dois modelos. Assim, usando o modelo proposto, além de apresentar acurácia de igual módulo, elimina todo o esforço computacional exigido pelo modelo iterativo. O esforço computacional na iteração de poucas dezenas de pontos (marcos geodésicos) pode parecer irrelevante, mas, em se tratando de milhões de pontos, como ocorre em grandes levantamentos (dados laser scanner, por exemplo), esse esforço torna-se considerável.

Embora não tenham sido realizados experimentos com os modelos diretos supracitados (PAUL, 1973; OZONE, 1985 e SOFAIR, 1993), por extrapolar o objetivo deste estudo, o tempo de processamento por aqueles modelos será maior que o esforço computacional demandado pelo método proposto, devido ao maior volume de cálculos intrínsecos às equações finais para determinação da Latitude. Maiores detalhes acerca 
desses métodos e comparações entre eles podem ser vistos em Gerdan e Deakin (1999), Fok e Iz (2003); Sofair (1993) e Burtch (2006).

$\mathrm{Na}$ execução dos experimentos apresentados, e de outros testes, foi observado que o programa Geocar apresenta falhas para valores de coordenadas cartesianas próximos a zero. O limiar, ou valor mínimo, para as coordenadas cartesianas de entrada no software é o,1 metro. Esse tipo de falha é comum em alguns softwares comerciais, que às vezes são elaborados sem o crivo de um profissional da área (Geodesista). Dessa forma, quanto mais simples for o modelo (direto), menor será a ocorrência desse tipo de erro.

Frente ao exposto neste texto, propõe-se aos desenvolvedores de softwares o uso intercalado dos dois modelos, em que o iterativo será usado apenas para conversão de coordenadas de pontos próximos ao equador, para latitudes menores que 1' (um minuto), em módulo, reduzindo, assim, o tempo de processamento e obtendo resultados mais acurados.

Este trabalho servirá de referência bibliográfica para pesquisadores e/ou profissionais que desejarem fazer uso ou implementar um modelo de transformação direta de coordenadas geodésicas em cartesianas.

\section{Referências Bibliográficas}

ALHANATI, L. S. Alfa Connection: Alfa Virtual School - Matemática - Elementos e nomenclatura da elipse. Disponível em: http://alfaconnection.net/pag_avsm/geoo501. htm. Acessoem: 28 de julho de 2011.

BURTCH, R. A comparison of methods used in rectangular to geodetic coordinate transformations. Anais... ACSM Annual Conference and Technology Exhibition, Orlando, April, 2006.

CLYNCH, J. R. Geodetic Coordinate Conversions. Disponível em: http:// www.gmat.unsw.edu.au/snap/gps/ clynch_pdfs/coordcvt.pdf. 2006.

DAL'FORNO, G. L.; AGUIRRE, A. J.; HILLEBRAND, F. L., GREGÓRIO, F. V. Transformação de coordenadas geodésicas em coordenadas no plano topográfico local pelos métodos da norma NBR 14166:1998 e o de rotações e translações.Simpósio Brasileiro de Ciências Geodésicas e Tecnologias da Geoinformação, 3. 2010, Recife. Anais...:Recife: SBCGTG, 2010. v.3. p.001-007. 
FEATHERSTONE, W. E.; CLAESSENS, S. J. Closed-form transformation between geodetic and ellipsoidal coordinates. StudyGeophysicsGeodetics, v. 52, 2008.

FOK, H. S.; IZ, H. B.A Comparative Analysis of the Performance of Iterative and Non-iterative Solutions to the Cartesian to Geodetic Coordinate Transformation. Journal of Geospatial Engineering, v. 5, n.2, 2003, p.61-74.

FRANCOIS, A. Sistemas de coordenadas y transformaciones. bases para eltrabajoen SIG. (Folheto Técnico), n.2, Febrero, 2000.

GEMAEL, C.; ANDRADE, J. B. Geodésia Celeste. Paraná: Ed. UFPR, 2004.389p.

GERDAN, G. P.; DEAKIN, R. E. Transforming Cartesian coordinates $\mathrm{X}, \mathrm{Y}, \mathrm{Z}$ to Geographical coordinates $\varphi, \lambda$, h. The Australian Surveyor, v. 44, n. 1, 1999, p. 55-63.

HEISKANEN, W. A.; MORITZ, H. Physical geodesy. United States of America: W. H. Freeman and Company, 1967.

MONICO, J. F. G. Posicionamento Pelo NAVSTAR-GPS: Descrição, Fundamentos e Aplicações. São Paulo: Ed. UNESP, 2000. 288p.

NADAL, C. A.Sistemas de Referência e Tempo em Geodésia. Departamento de Geomática, Politécnico, Universidade Federal do Paraná. 2006.

OZONE, M. I. Non-Iterative Solution of the $\varphi$ Equation. Surveying and Mapping, v. 45, n. 2, 1985, p. 169-171.

PAUL, M. K. A Note on Computation of Geodetic Coordinates from Geocentric (Cartesian) Coordinates. Bulletin Géodésique, n. 108, 1973, p. 134-139.

SÁ, N. C. Software GEOCAR V.1 - IAG/USP/2007. Disponível em: http://www.iag.usp.br/geofisica/geodesia/raiz/menu_geocar.htm. Acesso em: 07 de maio de 2011. 
SEEBER, G. Satellite Geodesy: Foundations, Methods and Aplications. Berlin, New York: Walter de Gruyter, 1993.356p.

SEEMKOOEI, A. A. Comparison of different algorithms to transform geocentric to geodetic coordinates. Survey Review. v. 36, n. 286, 2002, p.627-633.

SILVA, I. Curso de Geomática. Universidade de São Paulo, Escola de Engenharia de São Carlos / SP. 2011.

SOFAIR, I.A Method for Calculating Exact Geodetic Latitude and Altitude. Naval Surface Warfare Center. n. 85, 1993.

Submetido em: 28/07/2011

Aceito em: 14/11/2012 
